\title{
On a viscous critical-stress model of martensitic phase transitions
}

\author{
John Weatherwax ${ }^{\text {a) }}$ \\ Department of Mathematics, Massachusetts Institute of Technology, 77 Massachusetts Ave., Cambridge, \\ Massachusetts 02139-4307, USA \\ Dimitri Vaynblat \\ Department of Applied \& Computational Mathematics, California Institute of Technology, \\ 1200 East California Blvd., Pasadena, California 91125, USA \\ Oscar Bruno \\ Department of Applied \& Computational Mathematics, California Institute of Technology, \\ 1200 East California Blvd., Pasadena, California 91125, USA \\ Ruben Rosales \\ Department of Mathematics, Massachusetts Institute of Technology, 77 Massachusetts Ave., Cambridge, \\ Massachusetts 02139-4307, USA
}

(Received 26 February 2007; accepted 21 July 2007; published online 19 September 2007)

\begin{abstract}
The solid-to-solid phase transitions that result from shock loading of certain materials, such as the graphite-to-diamond transition and the $\alpha-\epsilon$ transition in iron, have long been subjects of a substantial theoretical and experimental literature. Recently a model for such transitions was introduced which, based on a CS condition (CS) and without use of fitting parameters, accounts quantitatively for existing observations in a number of systems [Bruno and Vaynblat, Proc. R. Soc. London, Ser. A 457, 2871 (2001)]. While the results of the CS model match the main features of the available experimental data, disagreements in some details between the predictions of this model and experiment, attributable to an ideal character of the CS model, do exist. In this article we present a version of the CS model, the viscous CS model (vCS), as well as a numerical method for its solution. This model and the corresponding solver results in a much improved overall CS modeling capability. The innovations we introduce include: (1) Enhancement of the model by inclusion of viscous phase-transition effects; as well as a numerical solver that allows for a fully rigorous treatment of both, the (2) Rarefaction fans (which had previously been approximated by "rarefaction discontinuities"), and (3) viscous phase-transition effects, that are part of the vCS model. In particular we show that the vCS model accounts accurately for well known "gradual" rises in the $\alpha-\epsilon$ transition which, in the original CS model, were somewhat crudely approximated as jump discontinuities. () 2007 American Institute of Physics. [DOI: 10.1063/1.2778634]
\end{abstract}

\section{INTRODUCTION}

Shock-induced solid-to-solid phase transitions have long been subjects of a substantial theoretical and experimental literature; prominent examples of such work include the contributions $^{1-3}$ on the $\alpha-\epsilon$ iron transition and graphite-todiamond transition. In these contributions, as in many others, shock waves in solids are induced through the impact of planar projectiles, leading to essentially planar fronts propagating through a flat slab. The phase transitions under consideration then manifest themselves through a rather peculiar phenomenon: upon impact, not one but two parallel shock fronts are induced in the slab.

Recently, a model for such shock-induced phase transitions (called "the CS model" in what follows) was introduced which, based on a critical stress (CS) condition and without use of fitting parameters, accounts quantitatively for existing observations in a number of systems. ${ }^{4}$ The main emphasis of earlier analysis lies on metastability and the kinetics of the transformation processes; see e.g. Refs. 5 and 6. The resulting models depend on a variety of material param-

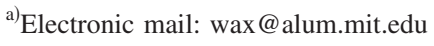

eters and functions which are difficult to obtain. As shown in Ref. 4, the major aspects of the shock-induced transition processes under consideration can in fact be captured without recourse to such detailed information. While the results of the CS model match quantitatively the main features of the available experimental data, disagreements in some details between the predictions of this model and experiment, attributable to an ideal character of the CS model, do exist; see Figs. 1 and 2. Note, in particular, the "gradual" rise times of the second shocks in the $\alpha-\epsilon$ iron transition, which the ideal CS model approximates, rather crudely, as jump discontinuities.

[Each curve in Figs. 1 and 2 represents a record of the free surface velocity for one experiment. The vertical axis is the free surface velocity and the horizontal axis is time. In terms of the impactor velocity, the curves order naturally from bottom (lowest impactor velocity) to top (highest impactor velocity). The curves in Fig. 1 are labeled by the impactor velocity, as in Ref. 3. The curves in Fig. 2, in turn, are labeled by experiment number, matching the enumeration used in Ref. 2. Only a few representative samples of the experimental curves are included in these plots.] 

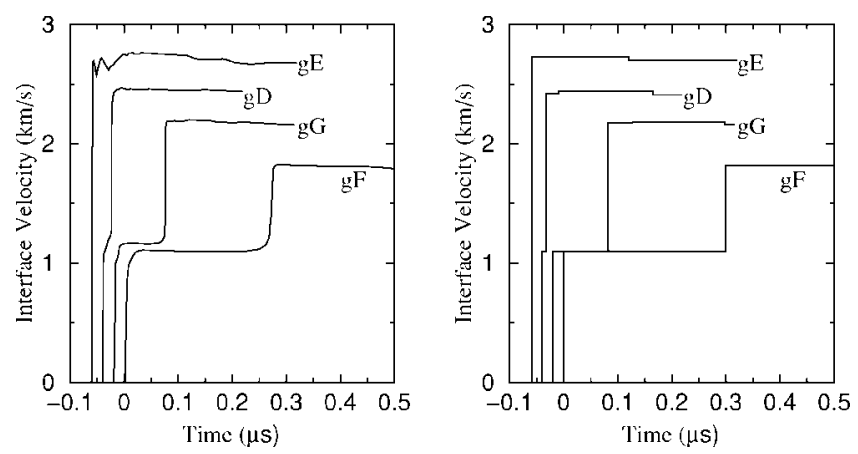

FIG. 1. Free surface velocity profiles for the graphite-diamond phase transition in shock loaded graphite. Left: Experimental measurements by Erskine and Nellis (Ref. 3). Right: The ideal CS model, using the rarefaction discontinuity approximation as described in Ref. 4.

In this article we present a version of the CS model, the viscous $\mathrm{CS}$ model (vCS), as well as a numerical method for its solution. This model and the corresponding solver results in a much improved overall CS modeling capability. The innovations we introduce include: (1) Enhancement of the model by inclusion of viscous phase-transition effects; as well as a numerical solver that allows for a fully rigorous treatment of both, the (2) rarefaction fans (which, in order to reduce the problem to a sequence of Riemann problems, had previously been approximated by "rarefaction discontinuities"), and the (3) viscous phase-transition effects, that are part of the vCS model. In particular we show that the vCS model accounts accurately for well known "gradual" rises in the $\alpha-\epsilon$ transition which, in the original CS model, were somewhat crudely approximated as jump discontinuities.

This article is organized as follows: after a review of the CS model (Sec. II), we present, in Sec. III, predictions arising from a numerical solver for the (nonviscous) CS model that, unlike that of Ref. 4, accounts accurately for rarefaction fans. Our viscous CS model is then introduced in Sec. IV. A correct numerical discretization of the dissipative phase transition is given in Sec. V. Numerical results arising from the viscous CS model together with relevant comparisons with experimental data (including a study of validity of the values of viscosity used) are given in Sec. VI; a number of conclusions we have drawn from this work, finally, are presented in Sec. VII.
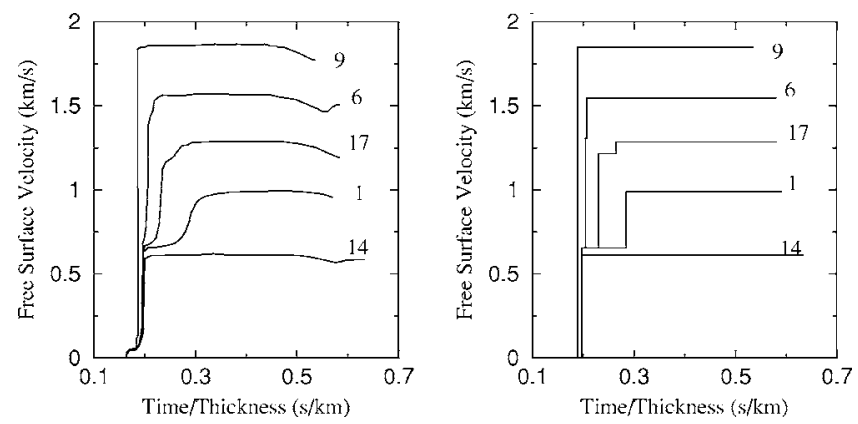

FIG. 2. Free surface velocity profiles for the $\alpha-\epsilon$ phase transition in shock loaded iron. Left: Experimental measurements by Barker and Hollenbach (Ref. 2). Right: The ideal CS model, using the rarefaction discontinuity approximation as described in Ref. 4.

\section{IDEAL CS MODEL}

We begin by listing the main assumptions implicit in the previous version of the CS model, which we will also refer to as the ideal CS model; full details can be found in Refs. 4 and 7 .

\section{A. Fundamental postulates}

The CS model, which describes the phase transitions under shock loading between a low density phase (LD) (e.g., the graphite phase in the graphite-diamond transition, or $\alpha$ phase in the $\alpha-\epsilon$ iron transition), and a high density phase (HD) (e.g., the diamond phase in the graphite-diamond transition, or $\epsilon$ phase in the $\alpha-\epsilon$ iron transition), is based on the following postulates:

(1) The target is a plate whose thickness is much smaller than its transversal dimensions, so that all measurements are completed before the arrival of any release wave originating at the lateral faces of the slab. This assumption allows one to assume that the slab is of infinite lateral extent, and that the motion is one dimensional, in the direction normal to the plate.

(2) In the graphite and iron systems under consideration, independently of whether stresses may be assumed to be hydrostatic (iron) or not (graphite, diamond), the equations of motion for each one of the pure phases, expressed in terms of the quantities $(v, q, u)$ (specific volume, negative normal stress, and velocity), are formally identical to the equations governing one-dimensional flow of an inviscid gas; see Ref. 4. Note that for hydrostatic stresses the negative normal stress $q=-\tau_{11}$ equals the pressure $p=-\left(\tau_{11}+\tau_{22}+\tau_{33}\right) / 3$.

(3) In each system the normal stress $q$ in the LD phase cannot exceed a (temperature dependent) critical value $q_{\text {crit }}^{\mathrm{LD}}(T)$. Similarly, the normal stress $q$ in the HD phase cannot lie below a (temperature dependent) critical value $q_{\text {crit }}^{\mathrm{HD}}(T)$. This is known as the CS postulate. In the hydrostatic cases considered in this article the pressure nomenclature will be used [see comment at the end of point (2) above], and the critical pressures for the forward and reverse transformations will be denoted by $p_{\text {crit }}^{\mathrm{LD}}(T)$ and $p_{\text {crit }}^{\mathrm{HD}}(T)$, respectively.

\section{B. Mathematical formulation}

From assumptions (1) and (2) in Sec. II A, the governing dynamical equations for the CS model reduce to onedimensional conservation of mass and momentum. ${ }^{8}$ In an Eulerian frame of reference these are

$$
\frac{\partial}{\partial t} \rho+\frac{\partial}{\partial x}(\rho u)=0 \quad \text { and } \quad \frac{\partial}{\partial t}(\rho u)+\frac{\partial}{\partial x}\left(\rho u^{2}+p\right)=0,
$$

where $\rho$ is the density, $u$ is the particle velocity, $p$ is the pressure, $t$ is the time, and $x$ is the Eulerian spatial position. An equivalent form, in a Lagrangian frame of reference is 


$$
\frac{\partial v}{\partial t}-\frac{\partial u}{\partial \xi}=0 \quad \text { and } \quad \frac{\partial u}{\partial t}+\frac{\partial p}{\partial \xi}=0,
$$

where $v \equiv 1 / \rho$ is the specific volume and $\xi$ is the Lagrangian spatial coordinate, related to the Eulerian coordinate $x$ by

$$
\xi=\int_{x_{0}}^{x} \rho(\widetilde{x}, t) \mathrm{d} \widetilde{x}
$$

where $x_{0}=x_{0}(t)$ is the Eulerian position of a fixed mass particle. An Eulerian coordinate system is used throughout this text.

From assumption (3) of the CS model (see Sec. II A), the empirical pressure function $p=p(v)$ has the discontinuous form

$$
p(v)= \begin{cases}p^{\mathrm{LD}}(v) & \text { for } p<p_{\text {crit }}^{\mathrm{LD}}, \\ p^{\mathrm{HD}}(v) & \text { for } p>p_{\text {crit }}^{\mathrm{HD}} .\end{cases}
$$

As specified in the CS theory we assume from now on that both $p_{\text {crit }}^{\mathrm{LD}}$ and $p_{\text {crit }}^{\mathrm{HD}}$ are known constants. In what follows an equation of state of the Mie-Grüneisen form

$$
p(\eta)=\frac{\rho_{0} c_{0}^{2} \eta}{(1-s \eta)^{2}}
$$

is assumed for both $p^{\mathrm{LD}}(v)$ and $p^{\mathrm{HD}}(v)$. Here $\eta$ is defined by

$$
\eta=1-\frac{\rho_{0}}{\rho}=1-\frac{v}{v_{0}},
$$

the values of the coefficients we use are those specified in Ref. 4.

When $p=p(v)$ is a convex smooth function with negative derivative, the system in (1) - equivalently, in (2) - is known as the $p$ system, and has been extensively studied in the mathematical literature, see Ref. 9. In the CS theory, because of the switch in the equation of state at the critical pressure, $p$ is neither convex nor continuous, and further assumptions are needed to have a complete theory. The mathematical consequences of this change in functional form of the equation of state are discussed fully in Ref. 4 . The main consequences of this particular equation of state over that of the $p$ system are that new waves, called critical transformation fronts, are introduced into the Riemann problem; see Ref. 4 for details.

\section{Ideal CS model: Results}

In this section we summarize some of the main results obtained under the CS hypothesis. ${ }^{4}$ Assuming the equation of state (4), Eq. (2) and the initial conditions

$$
\begin{aligned}
p(x, 0) & =\left\{\begin{array}{ll}
p^{l}=0 & \text { for } x<0, \\
p^{r}=0 & \text { for } x>0,
\end{array} \text { and } u(x, 0)\right. \\
& = \begin{cases}u^{l}=u_{\mathrm{flyer}} & \text { for } x<0, \\
u^{r}=0 & \text { for } x>0,\end{cases}
\end{aligned}
$$

the resulting initial value problem is a Riemann problem whose solutions are, for sufficiently small times, piecewise constant discontinuous waves. To integrate further in time a simplifying approximation that restricts the approximate so-

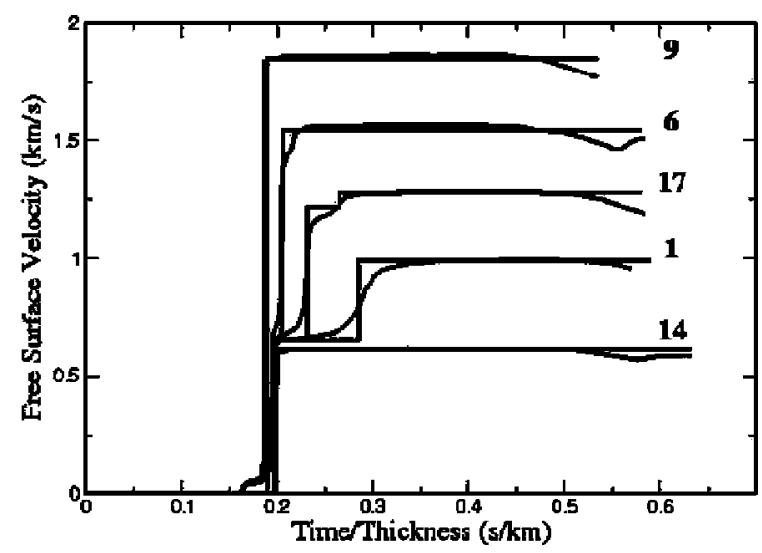

FIG. 3. A superposition of the experimental and theoretical free surface velocities (CS model under the rarefaction discontinuity approximation) for several of the $\alpha-\epsilon$ iron experiments presented by Barker and Hollenbach (Ref. 2).

lutions, for all times, to a class of piecewise constant discontinuous waves was then used. This approximation, which has been used for sometime in the solid mechanics community, is usually referred to as the "rarefaction discontinuity approximation." For comparisons used later in this article, in Fig. 3 we present a superposition of the experimental and theoretical predictions of the CS model under the rarefaction discontinuity approximation for several of the experiments presented by Barker and Hollenbach. ${ }^{2}$ The experiments shown in Fig. 3 represent the free surface velocity profiles for a range of impactor velocities in the $\alpha-\epsilon$ phase transition in shock loaded iron.

The agreement between experiment and the ideal CS theory for the iron experiments and under the CS model is significant, but of a lower quality than that obtained for the graphite system. Indeed, the two sets of curves shown in Fig. 2 differ in a number of ways:

(1) The theoretical free surface velocity profiles for the iron configurations are "too steep." The second wave in the experimental profiles numbered 1,17 , and 6 have a steep but finite slope (representing the increase in velocity achieved during the second wave). The predictions, however, give infinite rise times for the second wave in each experiment. The experimental curve numbered 1 has the shallowest rise time for the second wave, and correspondingly the worst match with theory.

(2) For long times the predicted free surface velocity profiles asymptote to constant values. Many of the experimental curves have free surface velocities, however, that are not constant for large values of time. This change in free surface velocity are due to edge-wave effects which are not taken into account in the present onedimensional theoretical context, see assumption 1 in Sec. II A, and will therefore not be further considered in this work.

(3) In the experimental plots the first large jump in free surface velocity is preceded by a relatively small jump before the time/thickness of $0.2 \mathrm{~s} / \mathrm{km}$, that is not present in the theoretical plots. This "toe" is caused by the plastic response of iron, while the remaining wave curves 
are produced for stresses well beyond the elastic/plastic boundary in iron. The CS theory only considers wave propagation occurring in this high-pressure regime and, therefore, the "plastic toe" will not be further considered in this text.

In view of these considerations we conclude that the main point of disagreement between the ideal CS theory and experiment is that described in point (1) above.

In order to account for and correct this disagreement we consider the main approximations made in the ideal CS model, namely, (a) The rarefaction discontinuity approximation, and (b) Absence of dissipative effects. As shown in Sec. III, the use of the rarefaction discontinuity approximation in the present contexts gives rise to very minimal errors indeed and we are thus lead to attribute the disagreements between the ideal CS theory and experiment to the lack of dissipative mechanisms in the CS model. A dissipative model is then proposed in Sec. IV which, through numerical simulations using numerical algorithms introduced later in this article, will be shown to overcome the difficulties mentioned above and yield close agreement with experimental results.

\section{CRITICAL STRESS MODEL WITH FULL ACCOUNT OF RAREFACTION FANS}

As mentioned in the previous section, the effect of the rarefaction-discontinuity approximation ought to be considered as a possible source of the disagreements between experiment and the ideal CS model. In this section we thus present a set of results produced under the CS theory but without use of this approximation. To produce these results we used full numerical solution of rarefaction fans; our results span the various impact regimes corresponding to the experimental setups of Barker and Hollenbach. ${ }^{2}$

The numerical flows presented below were produced by means of two numerical schemes:

(1) A Godunov-type initial value solver.

(2) A numerical scheme, called the characteristic tracking method, developed in the course of this work. The characteristic tracking method will be further described elsewhere; see Refs. 7 and 10.

While the characteristic tracking method gives rise to more accurate results than the Godunov-based scheme, the results arising from the two numerical schemes used are in general agreement-within the accuracy of the Godunov solver. We thus present results produced by the characteristic tracking method only, since this approach yields better resolution of the flow features. The figures presented in what follows resulted from numerical simulations based on actual material constants as indicated in Refs. 4 and 7. (A number of interesting mathematical issues arising in these simulations, which relate to interactions of compressive and expansive simple waves under the CS model, will be presented elsewhere. ${ }^{4,7}$ )

In Fig. 4 we present a plot similar to Fig. 2 but with full numerical resolution of rarefaction fans; note that these simulations do not involve adjustable parameters: they are the results of a fully consistent zero parameter CS model

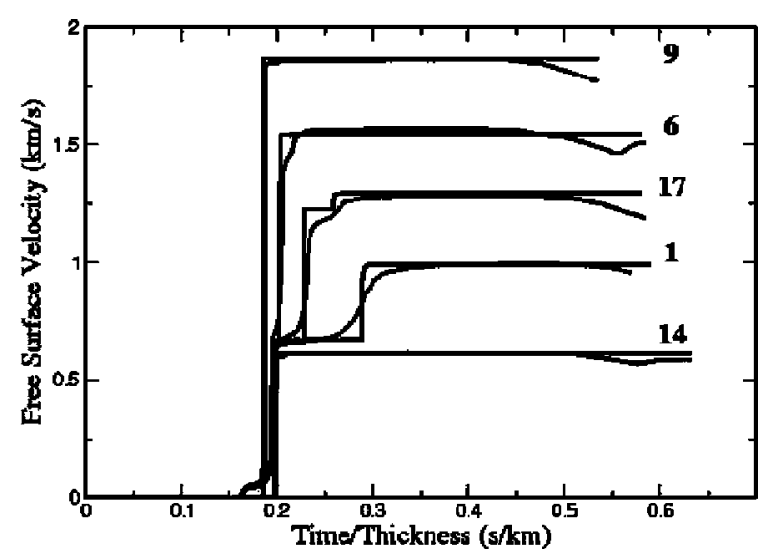

FIG. 4. Experimental and theoretical free surface velocity profiles for the $\alpha-\epsilon$ phase transition in shock loaded iron under the CS theoretical model, including full numerical resolution of rarefaction fans. We note that in profiles 1 and 17 the inclusion of rarefaction fans results in somewhat rounded corners of the free surface velocity plots but much of the vertical structure present in Fig. 3 still remains.

describing martensitic phase transformations. We note only slight differences between Figs. 2 and 4, and we thus conclude that the rarefaction approximation is not the main cause of the disagreements found between the CS theory and experiment for the iron system. In view of the discussion of the previous section we are now lead to attribute the disagreements between the ideal CS theory and experiment to the lack of account of dissipative phenomena within that model. In the following section we thus consider dissipative effects that may underlie the observed phenomena, and would need to be included in the CS model to produce more accurate simulations.

\section{THE VISCOUS CS MODEL (VCS)}

In view of the complex polycrystalline structure of iron, the large, approximately $20 \%$ volume contraction that accompanies the iron transitions should and does give rise to significant dissipative effects; in this section we incorporate dissipative terms in the our CS model, and we present numerical methods for the solution of the resulting equationswhich we refer to as the viscous CS model (vCS).

To include dissipation into our model we follow an approach similar to the one that is used in the modeling of dissipation by turbulent fluid flows: we add an "effective" viscosity to the governing equations-note that this viscosity is not molecular viscosity, which is usually quite small. This "viscosity" is a single free parameter for each one of the phases which, once determined, should be valid for all experimental configurations involving the given phase transition. In an experiment involving phase transitions and shocks in iron there will thus be two viscosity values: one associated with the dynamics of iron in the low density phase, and the other related to the high density phase. In practical terms, one viscosity value applies ahead of the precursor shock, and a second (as we will see, much larger one) behind it.

An important remark at this point is that the dissipative processes to be introduced should not affect the thickness of the transformation front itself: in line with the main concepts inherent in the ideal CS model, only two phases of iron exist 
in the experiments under consideration; therefore, there must be a sharp line separating the two phases-unless there are mixture regions, which we neglect in the present analysis. In particular, in our modified model, shocks will no longer be modeled as discontinuities, but the transformation fronts will still be discontinuity points-see Fig. 6 .

(An alternate approach to the issues under consideration is based on the concept of a "relaxation time." According to this concept phase transitions do not occur instantly-as assumed in the CS models. Models based on relaxations times have been used in the past (see for instance Refs. 5 and 6), and have in some cases been found to yield good agreement with smooth experimental phase-transition curves (see Fig. 2 ). It was also found that models based on relaxation-times necessitate, in order to achieve good agreement with experiment, a different relaxation time, determined from experiment, for each experimental curve. We propose that viscous effects are the more likely cause of the smoothing present in the velocity profiles and, in this article, we thus focus our efforts on studying the conclusions that result from such an assumption.)

In view of this discussion, the equations underlying the vCS model are, simply, the Eulerian Eq. (1) augmented to include viscosity

$$
\frac{\partial}{\partial t} \rho+\frac{\partial}{\partial x}(\rho u)=0, \quad \frac{\partial}{\partial t}(\rho u)+\frac{\partial}{\partial x}\left(\rho u^{2}+p\right)=\mu \frac{\partial^{2}}{\partial x^{2}} u,
$$

where the viscosity coefficient $\mu$ is a measure of the amount of dissipation associated with phase transitions and shocks. (As mentioned above two viscosity values should be used: one ahead of the precursor shock, and a second one behind it.) Equivalently, the Lagrangian Eq. (2) become

$$
\frac{\partial}{\partial t} v-\frac{\partial}{\partial \xi} u=0, \quad \frac{\partial}{\partial t} u+\frac{\partial}{\partial \xi} p=\mu \frac{\partial}{\partial \xi}\left(\frac{1}{v} \frac{\partial u}{\partial \xi}\right) .
$$

To study the effect that the added viscous term has on the free surface profiles, we solved numerically the Lagrangian Eq. (9); the result of these simulations are presented in Sec. VI. In the next section we present the numerical algorithm used in the solution of these equations; a discussion of the results obtained, finally, follows in Sec. VII.

\section{VISCOUS NUMERICS}

The natural division of the flow into separate domains, as discussed earlier, by a moving phase transformation discontinuity, will motivate the structure of the algorithm presented below-which we describe for rather general configurations, including, possibly more than one discontinuity and more than two domains. Specifically, we derive discrete numerical equations for $M$ domains, separated by $M+1$ interfaces-including the left end of the first domain and the right end of the last domain (which coincide with the leftand right-ends of the sample, respectively), see Fig. 5. It is fundamental to the vCS model (and hence the numerical algorithm that implements it) that in each domain there is only a single phase and a single equation of state. In particular, in each domain Eq. (9) has a unique meaning, and standard finite difference discretizations can be used on them. Our

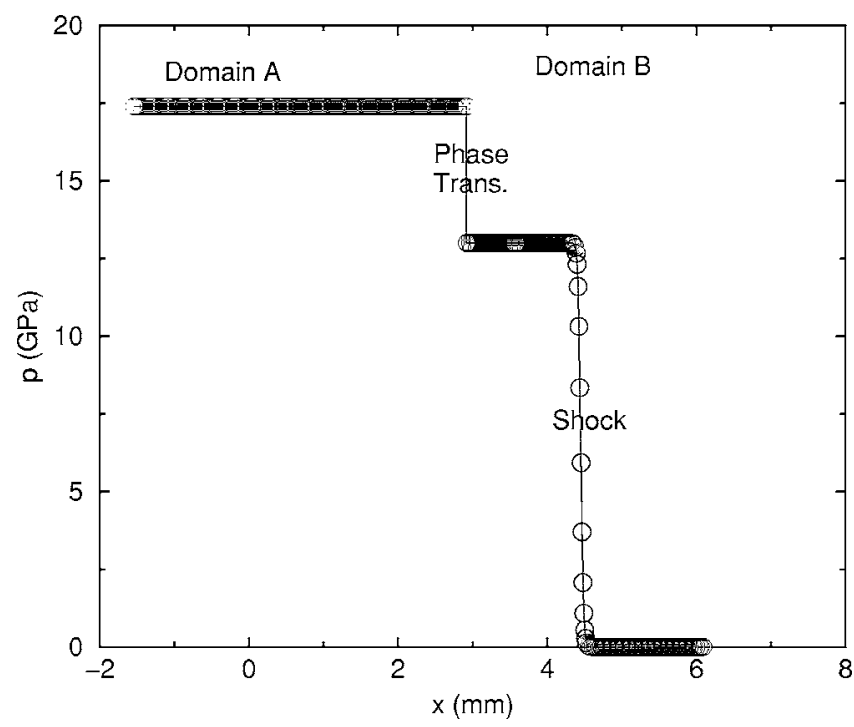

FIG. 5. Several domains separated by moving discontinuities, including the left end of the first domain and the right end of the last domain (which coincide with the left and the right end points of the sample, respectively). Each discontinuity is a phase transition, and in each domain there is a single phase.

integration problem will thus reduce to adequately solving the equations across the phase transition fronts, where the equation of state has a discontinuity and where, the significance of the spatial derivatives in Eq. (9) must be specified. Recognizing this fact, our numerical approach then relies on use of the method of lines to perform the time integration once a suitable spatial discretization is defined. At this point it is useful to outline the rest of Sec. V, which is the most technical part of this article. In Sec. V A we present a transformation of the independent variable $\xi$ that maps the individual constant phase domains (with variable end points) to a fixed stationary grid. In Sec. V A we then present the spatial discretization of our equations we use in the interior of each domain. In Sec. V B we present the discrete implementations we used across domain boundaries for the derivatives in Eq. (9). These implementations can be classified into several subcases depending on the type of the domain boundary under consideration; e.g., forward/backward transformation front, contact discontinuity, etc. With all spatial derivatives discretized, the numerical description can be completed by an appropriate temporal discretization; which is presented in Appendix C. Numerical results produced by means of the resulting algorithm are then presented in Sec. VI.

\section{A. Fixed-domain transformation and finite-difference spatial discretization}

Consider a section of the real line from $\xi_{L}$ to $\xi_{R}$, containing $M+1$ interfaces and $M$ domains, as in Fig. 5. Assume that, as a function of time $t$, the locations of the interfaces are given by the smooth functions $\xi=s_{i}(t)$, where $0 \leq i \leq M, \xi_{L}$ $=s_{0}(t)$, and $\xi_{R}=s_{M}(t)$ correspond to the left and right ends of the sample, respectively. Recalling that we work in Lagrangian coordinates, in what follows each one of the functions 
$\xi=s_{i}(t)$ are identified with the mass to the left of the corresponding interface: e.g., $\xi_{L}=0$, and $\mathcal{M}_{i}=\dot{s}_{i}(t)$ is the mass flux across the interface.

In each domain $s_{m-1}(t) \leq \xi \leq s_{m}(t)$ we define the coordinate transformation $\xi \rightarrow \xi_{m}$ by

$$
\xi_{m}=\frac{\xi-s_{m-1}(t)}{s_{m}(t)-s_{m-1}(t)}, \quad \text { so that }\left\{\begin{array}{l}
\xi=s_{m-1} \Leftrightarrow \xi_{m}=0, \\
\xi=s_{m} \Leftrightarrow \xi_{m}=1 .
\end{array}\right.
$$

Thus, the $m$ th domain is transformed into the fixed interval $[0,1]$ and the unknown functions $s_{m}(t)(1 \leq m \leq M)$ determine the positions of the corresponding interfaces. This type of transformation is commonly done in connection with calculations involving free boundaries. ${ }^{11}$ Within each domain we can reexpress Eq. (9) in terms of the local variable $\xi_{m}$ using Eq. (10); dropping the subscript $m$ in $\xi_{m}$ and the time dependence in $s_{m}(t)$, the equations thus become

$$
\frac{\partial}{\partial t} v-\frac{1}{s_{m}-s_{m-1}}\left[\left((1-\xi) \dot{s}_{m-1}+\xi \dot{s}_{m}\right) \frac{\partial}{\partial \xi} v+\frac{\partial}{\partial \xi} u\right]=0
$$

and

$$
\begin{gathered}
\frac{\partial}{\partial t} u-\frac{(1-\xi) \dot{s}_{m-1}+\xi \dot{s}_{m}}{s_{m}-s_{m-1}} \frac{\partial}{\partial \xi} u+\frac{1}{s_{m}-s_{m-1}} \frac{\partial}{\partial \xi} p \\
=\frac{\mu}{\left(s_{m}-s_{m-1}\right)^{2}} \frac{\partial}{\partial \xi}\left(\frac{1}{v} \frac{\partial u}{\partial \xi}\right) .
\end{gathered}
$$

We next discretize these equations using a finite difference staggered grid approach. In detail, we break up each domain $0 \leq \xi \leq 1$ into a uniform grid with $N$ nodes including the end points

$$
\xi_{i}=(i-1) \Delta \xi, \quad \text { where } \Delta \xi=\frac{1}{N-1} \quad \text { and } \quad 1 \leq i \leq N,
$$

we call the interval $\xi_{i} \leq \xi \leq \xi_{i+1}$ the ith cell-with center $\xi_{i+1 / 2}=\frac{1}{2}\left(\xi_{i}+\xi_{i+1}\right)$. Then, for the specific volume $v$ (and density $\rho=1 / v$ ) we use a node centered approach, in which the numerical scheme evolves the values $v\left(\xi_{i}, t\right)$ of the specific volume at the nodes. For the velocity, on the other hand, we use a cell centered approach: the numerical scheme evolves the values $u\left(\xi_{i+1 / 2}, t\right)$ of the velocity at the cell centers. It is now convenient to introduce notation to indicate whether a numerical variable is defined on nodes or cells: when referring to a cell centered variable, for example $u$, we will use a bar, as in $\bar{u}$; a node centered variable, on the other hand, will receive no special indication. Thus the notation $v_{\xi}$ applies to the node centered value of the $\xi$ derivative of the specific volume, while $\bar{v}_{\xi}$ means the cell centered value of the same quantity. With these definitions, we now write down the spatial discretization of the dissipative Eqs. (11) and (12) using the staggered grid technique. To update the conservation of mass equation in (11), we need node-centered discrete versions of the spatial derivatives in the equation. We thus use centered differences to approximate the spatial derivatives appearing in Eq. (11), i.e.,

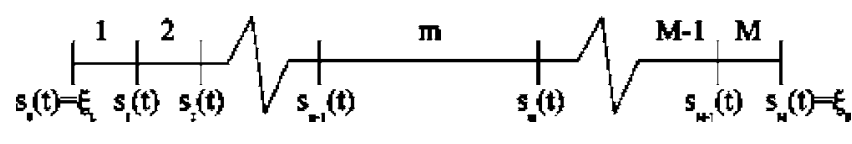

FIG. 6. Hypothetical diffusive pressure wave profile at a time shortly after impact. The marks in the horizontal axis measure distance, in millimeters, from the point of impact. The vertical marks measure pressure in Giga Pascals. Domain $A$ (respectively $B$ ) is defined as the region to the left (respectively, to the right) of the phase transformation. Here we see the rightfacing phase transformation, as a discontinuity, and the right-facing shock, slightly smeared by the viscosity, both propagating toward the free surface-located at $x \approx 6 \mathrm{~mm}$.

$$
\begin{aligned}
\frac{\partial}{\partial \xi} v\left(\xi_{i}, t\right) & \approx \frac{v\left(\xi_{i+1}, t\right)-v\left(\xi_{i-1}, t\right)}{2 \Delta \xi} \text { and } \frac{\partial}{\partial \xi} u\left(\xi_{i}, t\right) \\
& \approx \frac{\bar{u}\left(\xi_{i+\frac{1}{2}}, t\right)-\bar{u}\left(\xi_{i-\frac{1}{2}}, t\right)}{\Delta \xi} .
\end{aligned}
$$

When used in (11), the approximations (14), lead to the semidiscrete conservation-of-mass system

$$
\begin{aligned}
\frac{\partial}{\partial t} v\left(\xi_{i}, t\right)= & \left(\frac{\left(1-\xi_{i}\right) \dot{s}_{m-1}+\xi_{i} \dot{s}_{m}}{s_{m}-s_{m-1}}\right) \frac{v\left(\xi_{i+1}, t\right)-v\left(\xi_{i-1}, t\right)}{2 \Delta \xi} \\
& +\left(\frac{1}{s_{m}-s_{m-1}}\right) \frac{\bar{u}\left(\xi_{i+1 / 2}, t\right)-\bar{u}\left(\xi_{i-1 / 2}, t\right)}{\Delta \xi},
\end{aligned}
$$

which is second-order accurate in $\Delta \xi$. A similar procedure can be applied to the conservation of momentum Eq. (12); this equation requires time updates of the velocity, a cell centered variable, so that cell centered approximations for the various $\xi$ derivatives involved are needed. The exact discretizations used are presented in Appendix A. We next describe the spatial discretization used for noninterior (interface) points.

\section{B. The spatial discretization of the boundary conditions}

The discretizations for the conservation of mass and momentum equations in (15) and (C1) both require information about the specific volume and the velocity one node and one cell away from the cell or node on which the time derivative terms in the semidiscrete equations are centered: these equations cannot be used at points close to a boundary. We thus need to consider what happens at the end points of each domain, where two type of situations may arise: an end point may either coincide with the boundary of the sample (i.e.,: $s_{i}$ for $i=0$ or $i=M)$, or it may correspond to the location of a phase transition (i.e.,: $s_{i}$ for $0<i<M$ ). This leads to two different types of boundary conditions at end points which we call outer boundary conditions and inner boundary conditions, respectively. For convenience, we now restrict our discussion of boundary conditions to the two-domain case $M=2$, such as that depicted in Fig. 6-where a single rightmoving phase transformation front separates the high-density phase on the left from the low-density phase on the right. In this case, as indicated in the figure caption, we will call the high-density domain the domain $A$, and the low-density domain the domain $B$. In the next sections we present the supplemental equations used to solve for the nodes and cells 


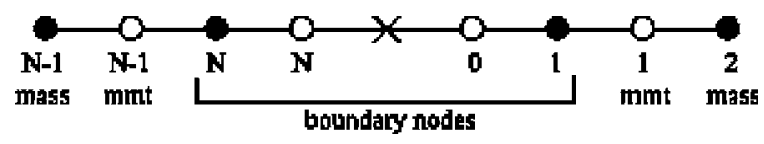

FIG. 7. Schematic representation of the numerical grid near an interface. The interface is indicated by a cross, the nodes by solid dots and the cell centers by open dots. The rightmost $(N$ th) node of the left domain, the leftmost (1st) node of the right domain, and the interface are the same point in space, but, since they play different roles in the numerical algorithm, they are represented separately in the diagram.

near the inner and outer boundary conditions mentioned earlier in this section.

\section{The outer boundary conditions}

The stencils of the semidiscrete equations in (15) and (C1) require the specification of variables one node and one cell to either side of the node or the cell at the stencil center. As a consequence of this fact, two boundary conditions are required on each outer boundary. In what follows we denote the left and right end points of the computational domain by $\xi_{L}=s_{0}$ and $\xi_{R}=s_{M}$.

Since we are not interested in computing for times so large that wave reflections from the back end of the impactor reach the free surface, we can assume that the left boundary of domain $A$ is at negative infinity. To model numerically the semi-infinite nature of domain $\mathrm{A}$ we then argue that, for a sufficiently large and negative value of $\xi$, the specific volume should be constant. Thus we set $v=v_{L}$ at $\xi=\xi_{L}$, where $v_{L}$ is obtained from the solution of the first Riemann problem (the collision of the impactor and the flyer), a calculation that is performed before the full viscous flow computation is started. This gives one of the two boundary conditions needed on the left end of the numerical domain. Similarly, we argue that on the right end of domain $B$ the specific volume should also be a constant, i.e.,: $v=v_{R}$ on $\xi_{R}$, where the constant is determined by the equation $p\left(v_{R}\right)=0$. This follows because the right boundary of domain $B$ is a free surface, and cannot support any pressure. Finally, to determine the second boundary condition required at each end, we use the conservation of mass equation in (9). From this equation it is clear that, if $v$ is constant along a particle path $\xi$ $=$ const, we must have

$$
\frac{\partial}{\partial \xi} u=0 .
$$

This gives a second boundary condition, applicable on any $\xi=$ const boundary where $v$ is kept constant.

\section{The inner boundary conditions}

Here we formulate the boundary conditions used at the interface between the two domains, $A$ and $B$, across which there is a jump in the equation of state. In detail, we consider all the unknowns/variables at or near the interface that cannot be updated by either of the equations in (15) or $(\mathrm{C} 1)$, and we systematically provide additional equations that can be used to update their values.

We begin by considering the immediate neighborhood of the interface and the associated unknowns. Figure 7 shows a schematic representation of the situation. In this figure the interface is denoted with a cross, the nodes with solid dots and the cell centers with open dots. There are some important clarifications that must be made with regard with this figure: the rightmost node of the left domain (node $N$ in the figure), the leftmost node of the right domain (node 1 in the figure), and the interface lie at the same point in space, but we have represented them separately in the figure because they play different roles in the numerical algorithm. Similarly, there is no cell $N$ belonging to the left domain, nor is there a cell 0 belonging to the right domain, but (for numerical reasons, which will become clear in our discussion below) these ghost cells have been introduced in the figure.

The representation of the numerical grid shown in Fig. 7 provides a convenient way to label the unknowns around the interface. In this representation, each node and cell center carries one unknown (the values of the specific volume and velocity, respectively) while the interface carries two unknowns, namely: its Lagrangian coordinate position $\xi=s(t)$, and the mass flow across the interface $\mathcal{M}=\dot{s}$-both as introduced by the transformation in (10), mapping the equations to a fixed $[0,1]$ grid.

To update (in time) the specific volume in the node labeled $N-1$, the formula in Eq. (15) can be used, since an additional cell and node exist to either side of this node. The same statement can be made about the cell center labeled $N-1$, using the formula in Eq. (C1). Similarly, the cell center labeled 1, and the node labeled 2, can be updated using the semi-discrete formulas in (15) and (C1). This still leaves six variables for which the regular stencil used in the semidiscrete equations cannot be used, and for which extra equations are needed, namely:

$$
v_{N}, \bar{u}_{N}, s, \dot{s}, \bar{u}_{0}, \text { and } v_{1} .
$$

The first extra equation is obtained by approximating the mass flux through the interface $(\mathcal{M}=\dot{s})$ by the finite difference formula

$$
\dot{s} \approx \frac{s^{n+1}-s^{n}}{\Delta t},
$$

where $s^{n}$ is the value of $s=s(t)$ at time $t=t_{n}$, and $\Delta t=t_{n+1}$ $-t_{n}$. The other five extra equations will depend on the type of interface between the two domains; briefly, two equations follow from the Rankine-Hugoniot jump conditions (conservation of mass and momentum across the interface), one equation from knowledge of the specific interface type, and the remaining two equations are obtained from a certain numerical extrapolation process to be described below. These extra equations are given in their continuous forms in what follows; the corresponding discrete expressions are presented in Appendix B. There are two cases to consider, depending on the interface type.

a. Case 1: the interface is a forward or backward transformation front Two of the equations follow from the conservation of mass and momentum across the interface, where care must be taken to include the fluxes due to the dissipative terms. Modifying the standard Lagrangian RankineHugoniot equations to include the fluxes due to dissipation, we obtain 


$$
\dot{s}[v]+[u]=0 \quad \text { and } \quad \dot{s}[u]-\left[p(v)-\frac{\mu}{v} \frac{\partial}{\partial \xi} u\right]=0,
$$

where the partial derivative $u_{\xi}$ must be computed without crossing the discontinuity-it is because of the need to compute this derivative that the ghost cells were introduced earlier. We note that, we have assumed that there is no viscous contribution to the momentum flux arising from the phase transformation. Mathematically this means that singularities of order higher than Dirac deltas are ignored. These singularities arise from the dissipative term in (9), because the variables are discontinuous across the interface. After this is done, the second jump condition above in (18) guarantees the cancellation of the remaining distribution (Dirac's deltas) part in the momentum equation in (9).

A further condition, that is specific to transformation fronts (forward and backward), follows from the fact that the pressure ahead of the wave must be at the critical value for the phase into which the wave propagates. This gives a third equation

$$
p_{N}=p_{\text {crit }}^{\{\mathrm{LD}, \mathrm{HD}\}} \quad \text { or } \quad p_{1}=p_{\text {crit }}^{\{\mathrm{LD}, \mathrm{HD}\}},
$$

where the specific formula used depends on the direction of propagation of the interface, and the type of the phase transformation.

Finally, the two remaining boundary conditions are obtained from a numerical approximation. As explained above, the ghost cells are introduced because of the need to compute the derivatives $u_{\xi}$ in the right equation in (18). However, this is meaningless unless values for the velocities are provided at the ghost cell centers. A reasonable approach to doing this is to use extrapolation of the values of the velocity, from the inside the domain the ghost cell belongs to. We found, through experimentation with various possibilities, that first order extrapolation gives reasonable results for this purpose. This yields, on the uniform grids we used [see (13)], the following equations for $\bar{u}_{N}$ and $\bar{u}_{0}$

$$
\bar{u}_{N}=2 \bar{u}_{N-1}+\bar{u}_{N-2} \quad \text { and } \quad \bar{u}_{0}=2 \bar{u}_{1}+\bar{u}_{2} .
$$

b. Case 2: the interface is an interior contact discontinuity In this case

$$
\frac{d s}{d t}=0,
$$

because no mass crosses the interface. This gives a condition specific to contact discontinuities only. Conservation of mass and momentum now take the simpler forms

$$
[u]=0 \quad \text { and } \quad\left[p(\mathrm{v})-\frac{\mu}{\mathrm{v}} \frac{\partial}{\partial \xi} u\right]=0,
$$

where the same remarks made after the equations in (18) apply. Finally, two additional (numerical) boundary conditions are obtained by manipulation of the conservation of mass differential equation in (11). This equation yields, when evaluated on the $N$ th node

$$
\frac{d}{d t} v_{N}-\frac{1}{s-\xi_{L}} \frac{\partial}{\partial \xi} \bar{u}_{N}=0,
$$

where we have used the fact that we have only two domains, that the node is actually a particle path so that $\dot{s}=0$, and that the cell $N$ and the node $N$ are the same point in space (so that the node centered and cell centered derivatives are equivalent). A semidiscrete equation for $v_{N}$ can now be obtained by discretizing the spatial derivative of the velocity in this equation, using finite differences. This gives

$$
\frac{d}{d t} v_{N}-\frac{1}{s-\xi_{L}} \frac{3 \bar{u}_{N}-4 \bar{u}_{N-1}+\bar{u}_{N-2}}{2 \Delta \xi}=0,
$$

where the velocity value at the center of the ghost cell (i.e.,: $\bar{u}_{N}$ ) follows by the same extrapolation process used earlier in Eq. (20). A similar procedure generates a semi-discrete equation for $v_{1}$.

In Appendix B we present a detailed summary of all the semidiscrete equations used to update each node, cell, and interface (i.e.: $s$ ) at the boundary between domains. With this information, the numerical scheme is completely defined once we specify how to discretize the time derivatives. This is done with the rather well known " $\theta$ method" and as fully described in Appendix C. In the next section, we describe the results obtained using this numerical model.

\section{VISCOUS NUMERICAL RESULTS}

From the discussion in Sec. III we see that at least three different effective viscosity values may be relevant in our context: $\mu_{1}$ (relatively low) in the low-density phase ahead of the precursor shock, $\mu_{2}$ (relatively high) in the critical low-density phase behind the precursor shock, and $\mu_{3}$ in the high-density phase, behind the phase transformation. The dominant effect associated with the broadening of the second rise in the free surface experimental velocity plots, however, occur in the critical low-density phase region (where $\mu=\mu_{2}$ ), as the waves reflected back from the free surface (produced when the precursor shock strikes this interface) propagate into this region and interact with the phase transformation front. For the range of experimental times considered here, waves propagating in the material with viscosity $\mu_{3}$ will not have time to influence the measured free surface profile and are ignored (removing the need to explicitly model this value).

In the theoretical idealization of this two-viscosity model, $\mu=\mu_{1}$ before the precursor shock passes, after which it jumps to $\mu_{2}$. Rather than attempt to understand the physics and numerics of a viscous shock separating two different viscosities (which could be an entire research topic in its own right) what we do is much simpler while still retaining all the important modeling ideas. To study the effect that viscosity has on the free surface profiles, a simplified viscosity approach is taken: we set the viscosity $\mu$ in Eq. (9) to a spatially dependent function that continuously approximates a Heaviside step function representing the transition in viscosity between $\mu_{1}$ and $\mu_{2}$. Specifically, the functional form for $\mu$ is taken to be a hyperbolic tangent positioned horizontally in such a way that the transition region between viscosi- 

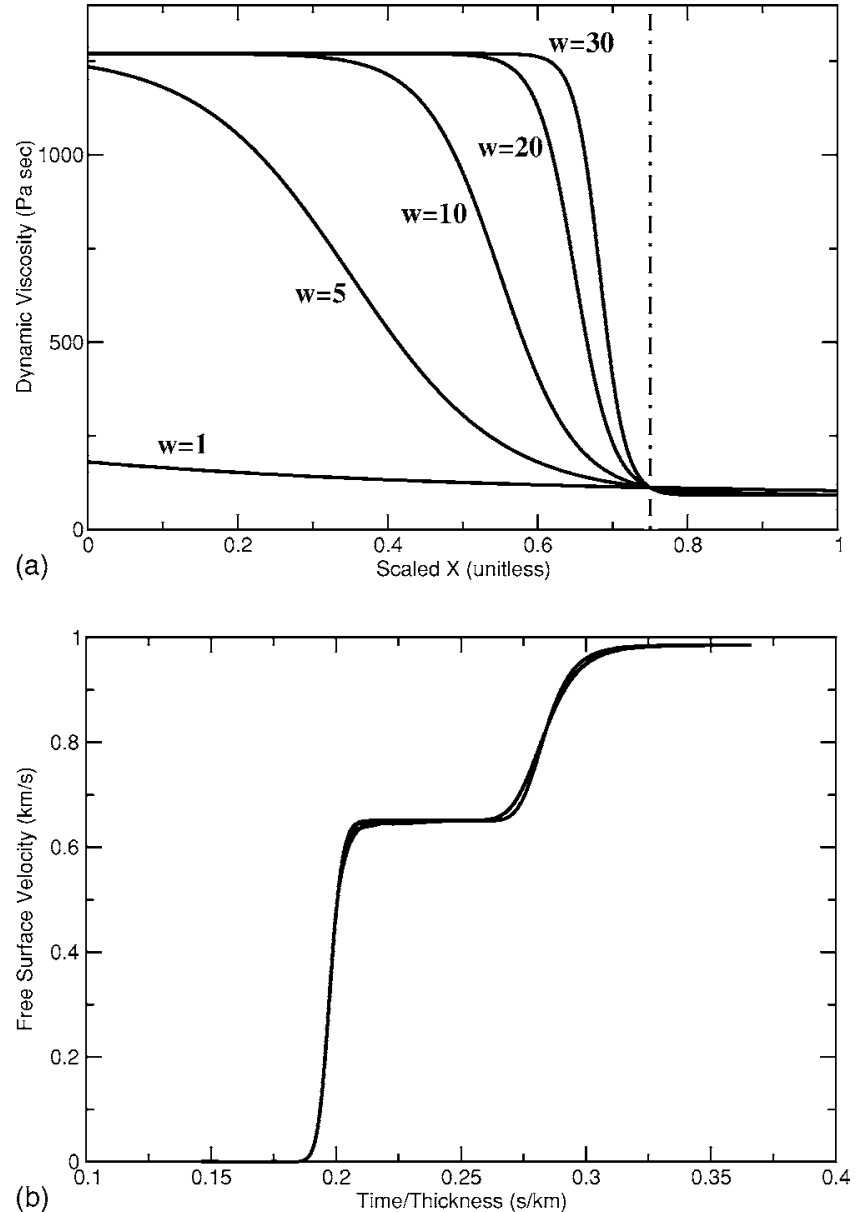

FIG. 8. Studies on the rate of transition between viscosities. Left: Plots of the spatial viscosity profiles obtained using a hyperbolic tangent transition. The hyperbolic tangent has a parameter $w$ which determines the rate at which $\mu_{1}$ changes to $\mu_{2}$. Larger values of $w$ make the viscosity change more rapid and smaller values of $w$ make the viscosity change more gradual. Shown in this picture are a sequence of $w$ values 1, 5, 10, 20, and 30 for an inviscid precursor shock located at $x=0.75$ (labeled with a dot-dashed line). Right: The free surfaces, for the first experiment in the Barker and Hollenbach paper, that result from using a hyperbolic tangent transition function with transition region ( $w$ ) given numerically by each of the numbers above.

ties occurs after the inviscid precursor shocks location. The explicit functional form used is given by

$$
\mu(x, t)=\frac{1}{2}\left(\mu_{2}-\mu_{1}\right)(1-\tanh (w(x-s t)+2))+\mu_{1},
$$

where $w$ is a "transition rate" parameter determining how quickly the transition between $\mu_{1}$ and $\mu_{2}$ takes place, and $s$ is the speed of the inviscid precursor shock as obtained from solving the initial Riemann problem according to the inviscid theory. A plot of this function for various transition rate parameters $(w)$ and $s=0.75$ appears in Fig. 8 . We found through experimentation that the free surface profiles for differing values of $w$ were almost identical (see Fig. 8) and for the remaining numerical experiments in this paper $w=20$.

The range of numerical experiments we conducted sought to find adequate viscosity values that best fit with the experimental data. It is important to point out that, with all assumptions previously stated, the same two viscosity values were usedto simultaneously fit all of the experimental curves,
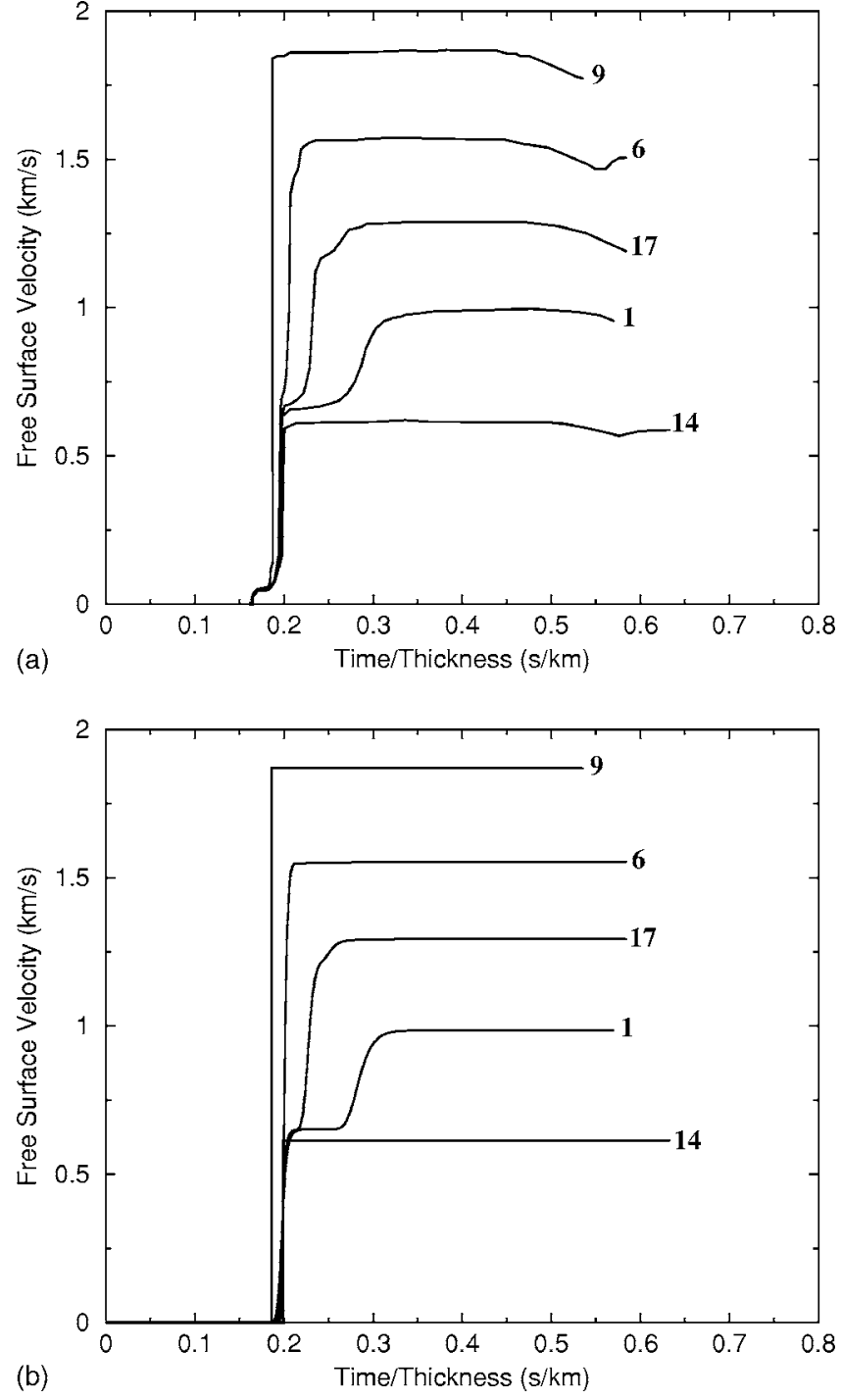

FIG. 9. Comparison between the Barker and Hollenbach experiments and the numerical solutions to the two-viscosity model, where the values of the two viscosities (the same for all the curves) are selected to optimize the fit. The actual viscosity values used are given in Sec. VI A.

so that the good fits we obtained (see Fig. 9) are not merely a consequence of using large numbers of free parameters. (For reference, in Sec. VI A below we provide some comparisons between the viscosity values we used and those obtained from both, molten iron, and from shock experiments in solid iron and aluminum without phase transitions.)

The arguments in Sec. III suggest that the "first" viscosity (the one valid in the sample ahead of the precursor shock) should be low. This is consistent with the experimental results: this viscosity value determines how spread-out the precursor shock is, and the first wave in the experimental plots is very sharp. It is only the second wave that is broad, which agrees with the idea that it is the phenomena that occur behind the precursor shock that are highly dissipative-due to the effects of the phase transition on the polycrystalline structure of the sample, as discussed in Sec. III.

The results of our investigations are summarized in Fig. 9, which shows a remarkably good agreement of theory and experiment. To find the values of the two viscosities we pro- 
ceeded as follows: we first found the value of the first viscosity, to have the best fit between calculations and experiments for the first wave. Then the second viscosity value was obtained so as to have the best fit with the second wave. We point out that the results shown are rather insensitive to the value of the first viscosity: we obtain good agreement with experiment as long as this value is sufficiently small. The sensitivity to the second viscosity is not large either: larger or smaller values by factors of up to two or three do not result in significant differences in the final results; the order of magnitude of the second viscosity is pinned down with certainty, however.

\section{A. Viscosity value}

The values of the two effective viscosities leading to the best fit curves shown in Fig. 9, calculated using the process described in Sec. VI, are

$$
\left.\begin{array}{l}
\mu_{1}=9.11 \times 10^{1} \mathrm{~Pa} \mathrm{~s}=9.11 \times 10^{2} \mathrm{P}, \\
\mu_{2}=1.27 \times 10^{3} \mathrm{~Pa} \mathrm{~s}=1.27 \times 10^{4} \mathrm{P},
\end{array}\right\}
$$

where the unit used is the cgs unit for dynamic viscosity, the Poise. Note that

$$
1 \mathrm{P}=1 \frac{\mathrm{g}}{\mathrm{cm} \mathrm{s}}=0.1 \frac{\mathrm{kg}}{\mathrm{m} \mathrm{s}}=0.1 \mathrm{~Pa} \mathrm{~s}
$$

and, for comparison, for water under standard conditions: $\mu \approx 10^{-2} \mathrm{P}$. It is also important to note that, while the values in (25) are the exact ones used to obtain the curves in Fig. 9, these curves are not very sensitive to the particular values used-as pointed out at the end of Sec. VI.

Obviously, we can interpret the process leading to (25) as that of providing a rough measurement of the effective viscosity-or dissipation coefficient-under the conditions of a large dynamic load produced by the precursor shock in the experiments by Barker and Hollenbach. The natural question is, of course, are these numbers reasonable? Is there agreement with other measurements, under similar conditions, to be found in the scientific literature? Clearly, there is no problem with the value of $\mu_{1}$ we obtained. This value should be interpreted as merely an upper bound on what the two-viscosity model requires for agreement with experiments, and the value is quite consistent with the values of viscosity for most fluids under normal conditions (i.e., these values do not exceed $\mu_{1}$ above). The real question has to do with the value of $\mu_{2}$, which is actually quite large (six orders of magnitude larger than the viscosity of water, for example). We consider this issue next.

Unfortunately, due to the extreme pressures experienced by the sample, direct measurements of the viscosity under these conditions would be very difficult, and we were unable to find any such results within the published literature. The only form of iron under pressures comparable to the ones studies here discussed in the literature, is molten iron at high pressure. The comparisons we provide in these regards are merely intended for comparisons of the orders of magnitude involved in the values of these quantities.

In molten iron at the high pressures involved, experimental measurements (again) would be very difficult to pur- sue, and we found none published. But theoretical calculations have been performed by a number of authors, mostly motivated by geophysical applications: the Earth's core is believed to be mostly composed of molten iron at a pressure about 10 times as large as the critical pressure for the $\alpha-\epsilon$ phase transformation we have considered in this text. ${ }^{12} \mathrm{~A}$ determination of the viscosity of the molten core is needed, for example, to explain the damping of radial seismic wave modes propagating in the earth. ${ }^{13}$ It is thus interesting to compare the value of the (second) viscosity $\mu_{2}$ we obtained, with the theoretical predictions from the geophysical literature.

A thorough literature review of published dynamical viscosity calculations for the Earth's core is presented in Ref. 14. The values reported there for the viscosities in the outer core span 14 orders of magnitude, from $10^{-2} \mathrm{P}$ on the lower end, to $10^{12} \mathrm{P}$ on the upper end. This huge variation is due, in part, to the varying number of ways used to obtain this parameter, most of which are very indirect (and probably not too reliable). The approaches include (for example) extrapolation from values measured at lower pressures, measurements based on the changes in the Earth's magnetic field over geological time scales, molecular dynamical simulations, and others. The value of $\mu_{2}$ reported in (25) lies in the middle of this wide range.

A second source of measurements, that we can use as a comparison check with our value for the second viscosity, is provided by the measurements of effective viscosities based on shock propagation experiments (without phase transitions) in solids. ${ }^{15-18}$ For example, Band ${ }^{15}$ used the approach of comparing the analytic viscous profile for a shock wave with the experimental profile, to obtain a value for the viscosity. Typical values for the viscosities obtained by these approaches are exemplified by the values (for aluminum) given by Prieto et al. ${ }^{16}$ at $1.4 \times 10^{3} \mathrm{P}$, and by Sakharov et al. ${ }^{17}$ at $2 \times 10^{4} \mathrm{P}$.

\section{CONCLUSIONS}

The ideal CS model, and the enhanced version introduced in this article, the viscous CS model, provide simple, yet effective and accurate, approaches for modeling of phase transformations under shock loading. In this article we demonstrated that the rarefaction discontinuity approximation, which had been previously utilized as an integral part of the ideal CS model, does not give rise to significant modeling errors. Further, we demonstrated that addition of a viscous term to the CS model produces the needed corrections to the ideal CS model to produce highly accurate modeling of the physics of iron phase-transitions under shock loading.

\section{APPENDIX A: DISCRETIZATION OF THE MOMENTUM EQUATION}

In this appendix we present the semidiscrete equations for the conservation of momentum, used in the numerical solution of the model for shock induced phase transitions, as modified by the addition of dissipation-see Sec. V A. We use the following cell centered approximations for the first order space derivatives that appear in Eq. (12), which follow from using centered differences: 


$$
\begin{aligned}
\frac{\partial}{\partial \xi} \bar{u}\left(\xi_{i+1 / 2}\right) & \approx \frac{\bar{u}\left(\xi_{i+3 / 2}, t\right)-\bar{u}\left(\xi_{i-1 / 2}, t\right)}{2 \Delta \xi} \text { and } \frac{\partial}{\partial \xi} p\left(\xi_{i+1 / 2}, t\right) \\
& \approx \frac{p\left(\xi_{i+1}, t\right)-p\left(\xi_{i}, t\right)}{\Delta \xi} .
\end{aligned}
$$

Similarly, for the viscous term we use the spatial discretization

$$
\begin{aligned}
\overline{\frac{\partial}{\partial \xi}\left(\frac{1}{v} \frac{\partial u}{\partial \xi}\right)}\left(\xi_{i+1 / 2}\right) \approx & \frac{1}{\Delta \xi^{2}}\left[\frac{\bar{u}\left(\xi_{i+3 / 2}, t\right)-\bar{u}\left(\xi_{i+1 / 2}, t\right)}{v\left(\xi_{i+1}, t\right)}\right. \\
& \left.-\frac{\bar{u}\left(\xi_{i+1 / 2}, t\right)-\bar{u}\left(\xi_{i-1 / 2}, t\right)}{v\left(\xi_{i}, t\right)}\right] .
\end{aligned}
$$

In all cases the terms on the right hand side of the expressions are defined in their natural domains (node centered densities and cell centered velocities). With these approximations, the semidiscrete equation for the conservation of momentum (correct up to second order in $\Delta \xi$ ) is then

$$
\begin{aligned}
\frac{\partial}{\partial t} \bar{u}\left(\xi_{i+1 / 2}, t\right)= & \left(\frac{\left(1-\xi_{i}\right) \dot{s}_{m-1}+\xi_{i} \dot{s}_{m}}{s_{m}-s_{m-1}}\right) \frac{\bar{u}\left(\xi_{i+3 / 2}, t\right)-\bar{u}\left(\xi_{i-1 / 2}, t\right)}{2 \Delta \xi} \\
& -\left(\frac{1}{s_{m}-s_{m-1}}\right) \frac{p\left(\xi_{i+1}, t\right)-p\left(\xi_{i}, t\right)}{\Delta \xi} \\
& +\frac{\mu}{\left(s_{m}-s_{m-1}\right)^{2}} \frac{1}{\Delta \xi^{2}}\left[\left(\frac{\bar{u}\left(\xi_{i+3 / 2}, t\right)-\bar{u}\left(\xi_{i+1 / 2}, t\right)}{v\left(\xi_{i+1 / 2}, t\right)}\right)\right. \\
& \left.-\left(\frac{\bar{u}\left(\xi_{i+1 / 2}, t\right)-\bar{u}\left(\xi_{i-1 / 2}, t\right)}{v\left(\xi_{i}, t\right)}\right)\right] .
\end{aligned}
$$

\section{APPENDIX B: SUMMARY OF THE INTERFACE BOUNDARY CONDITIONS}

In Sec. V a numerical algorithm was developed for the viscous Lagrangian conservation of mass and momentum Eq. (9), for a region containing several domains separated by moving discontinuities. In Sec. V B, boundary conditions were derived for the nodes and cells around the interface between two domains. Here we summarize the semidiscrete and fully discrete equations used at each node and cell near the interface. See Fig. 7 for the notation of the cell and node centered values used here. As we did in the body of this article, for simplicity we consider here the case where the forward transformation front is right facing while the backward transformation front is left facing. In this case we present a summary of the equations used for each variable around the interface, written as a system of equations.

(1) If the interface is a forward transformation front:

- Update $v_{N}$ using a discretization of the conservation of mass jump condition (18)

$$
\dot{s}\left(\mathrm{v}_{N}-\mathrm{v}_{1}\right)+\left(\frac{1}{2}\left(\bar{u}_{N}+\bar{u}_{N-1}\right)-\frac{1}{2}\left(\bar{u}_{0}+\bar{u}_{1}\right)\right)=0 .
$$

- Update $\bar{u}_{N}$ using first order extrapolation of velocity (20)

$\bar{u}_{N}-2 \bar{u}_{N-1}-\bar{u}_{N-2}=0$.
- Update $s$ using a discretization of the conservation of momentum jump condition (18)

$$
\begin{gathered}
\dot{s}\left(\frac{1}{2}\left(\bar{u}_{N}+\bar{u}_{N-1}\right)-\frac{1}{2}\left(\bar{u}_{0}+\bar{u}_{1}\right)\right)-\left(p\left(v_{N}\right)-p\left(v_{1}\right)\right) \\
+\frac{\mu}{\Delta \xi}\left(\frac{\bar{u}_{N}-\bar{u}_{N-1}}{v_{N}}-\frac{\bar{u}_{1}-\bar{u}_{0}}{v_{1}}\right)=0 .
\end{gathered}
$$

- Update $\bar{u}_{0}$ using first order extrapolation of velocity (20)

$\bar{u}_{0}-2 \bar{u}_{1}-\bar{u}_{2}=0$.

- Update $v_{1}$ using the critical condition (19)

$p\left(v_{1}\right)-p_{\text {crit }}^{\mathrm{LD}}=0$.

(2) If the interface is a contact discontinuity:

- Update $v_{N}$ using the conservation of mass partial differential Eq. (23), in domain $A$ evaluated at the local node $\xi=1$

$$
\frac{d}{d t} v_{N}-\frac{1}{s-\xi_{L}}\left(\frac{3 \bar{u}_{N}-4 \bar{u}_{N-1}+\bar{u}_{N-2}}{2 \Delta \xi}\right)=0 .
$$

- Update $\bar{u}_{N}$ with the conservation of mass jump condition (22)

$$
\frac{1}{2}\left(\bar{u}_{N}+\bar{u}_{N-1}\right)-\frac{1}{2}\left(\bar{u}_{0}+\bar{u}_{1}\right)=0 .
$$

- Update $s$ with (21)

$$
\frac{d s}{d t}=0 .
$$

- Update $\bar{u}_{0}$ with the conservation of momentum jump condition (22)

$$
-\left(p\left(v_{N}\right)-p\left(v_{1}\right)\right)+\frac{\mu}{\Delta \xi}\left(\frac{\bar{u}_{N}-\bar{u}_{N-1}}{v_{N}}+\frac{\bar{u}_{1}-\bar{u}_{0}}{v_{1}}\right)=0 .
$$

- Update $v_{1}$ using the conservation of mass partial differential Eq. (11) in domain $B$, evaluated at the local node $\xi=0$ with the one sided differences

$$
\frac{d}{d t} v_{1}-\frac{1}{\xi_{R}-s}\left(\frac{-3 \bar{u}_{1}+4 \bar{u}_{2}-\bar{u}_{3}}{2 \Delta \xi}\right)=0 .
$$

(3) If the interface is a backward transformation front:

- Update $v_{N}$ with the critical pressure condition (19) $p\left(v_{N}\right)-p_{\text {crit }}^{\mathrm{HD}}=0$.

- Update $\bar{u}_{N}$ with first order extrapolation of velocity (20)

$\bar{u}_{N}-2 \bar{u}_{N-1}-\bar{u}_{N-2}=0$.

- Update $s$ using the conservation of momentum jump conditions (18) 


$$
\begin{gathered}
\dot{s}\left(\frac{1}{2}\left(\bar{u}_{N}+\bar{u}_{N-1}\right)-\frac{1}{2}\left(\bar{u}_{0}+\bar{u}_{1}\right)\right)-\left(p\left(v_{N}\right)-p\left(v_{1}\right)\right) \\
+\frac{\mu}{\Delta \xi}\left(\frac{\bar{u}_{N}-\bar{u}_{N-1}}{v_{N}}-\frac{\bar{u}_{1}-\bar{u}_{0}}{v_{1}}\right)=0 .
\end{gathered}
$$

- Update $\bar{u}_{0}$ using first order extrapolation of the velocity (20)

$\bar{u}_{0}-2 \bar{u}_{1}-\bar{u}_{2}=0$.

- Update $v_{1}$ using conservation of mass jump condition (18)

$$
\dot{s}\left(v_{N}-v_{1}\right)+\left(\frac{1}{2}\left(\bar{u}_{N}+\bar{u}_{N-1}\right)-\frac{1}{2}\left(\bar{u}_{0}+\bar{u}_{1}\right)\right)=0 .
$$

With these specifications, the numerical scheme is well defined and can be implemented. Care must be taken, of course, to ensure that, when the interface changes type, these changes are detected and the correct equations are used.

\section{APPENDIX C: DISCRETIZATION OF THE TIME DERIVATIVES}

In this appendix, using the method of lines approach, we complete the construction of the viscous numerical algorithm. We do so by presenting the strategy used to discretize the time derivatives in the semidiscrete equations derived in the earlier sections. To this end we use the $\theta$ method, which leads to some of the most commonly used (simple) algorithms for time integration.

The semidiscrete equations for the dissipative model can be written in the compact vector form for the resulting ordinary differential equations (odes)

$$
\frac{d}{d t} Y=f(Y)
$$

where $Y=Y(t)$ is a vector representing the solution to the problem, and $f$ is the nonlinear vector function that follows upon writing the semidiscrete equations in terms of $Y$. Specifically, we can write $Y$ as a vector having one block of entries per domain, with the blocks separated by the variable giving the position of the corresponding interface $s_{n}$. Furthermore, within each block let the $Y$ entries alternate between the node centered specific volume values $v_{j}$ and the cell centered flow velocity values $\bar{u}_{j+1 / 2}$. Note that the values of $u$ in the ghost cells are not included in $Y$, since they are not evolved in time, and are computed directly by the purely algebraic equations in (20).

Let now $0 \leq \theta \leq 1$ be a parameter, whose value can be used to control the accuracy and stability of the algorithm (see below). Introduce also a time discretization, with $t_{n+1}$ $=t_{n}+\Delta t$ and $Y^{n}=Y\left(t_{n}\right)$. Then the $\theta$-method algorithm for the ode in (C1) is given by

$$
F\left(\Delta Y^{n}, Y^{n}\right) \equiv \Delta Y^{n}-\Delta t f\left(Y^{n}+\theta \Delta Y^{n}\right)=0,
$$

where $\Delta Y^{n}=Y^{n+1}-Y^{n}$, and $F$ is defined by the formula. It is well known that: when $\theta \geq 1 / 2$ this algorithm is unconditionally stable, and when $\theta<1 / 2$ the algorithm is only conditionally stable. ${ }^{19}$ Particular values of $\theta$ give rise to some common time integration strategies:

- $\theta=0$ gives the explicit forward Euler scheme.

- $\theta=\frac{1}{2}$ gives the second order, centered implicit trapezoidal rule (or Crank-Nicholson).

- $\theta=1$ leads to the backward implicit Euler method.

In our calculations we used a value of $\theta$ slightly above $1 / 2$ (in fact $\theta=0.55$ ), to assure maximum stability and accuracy.

Given the current state $Y^{n}$, Eq. (C2) is a (generally nonlinear) system of equations for the increment vector $\Delta Y^{n}$, which we solve using Newton's method. This requires the calculation of the Jacobian of the nonlinear vector function $F$, which is a cumbersome but straightforward task-since, in fact, $F$ is made up by fairly simple formulas. Since the equations used at the interface change as the interface changes type, we monitor when this change take place, and then adjust the formulas for the function $F$ and its Jacobian accordingly.

${ }^{1}$ D. Bancroft, E. L. Peterson, and S. Minshall, J. Appl. Phys. 27, 291 (1956).

${ }^{2}$ L. M. Barker and R. E. Hollenbach, J. Appl. Phys. 45, 4872 (1974).

${ }^{3}$ D. J. Erskine and W. J. Nellis, J. Appl. Phys. 71, 4882 (1992).

${ }^{4}$ O. Bruno and D. Vaynblat, Proc. R. Soc. London, Ser. A 457, 2871 (2001).

${ }^{5}$ J. C. Boettger and D. C. Wallace, Phys. Rev. B 55, 2840 (1997).

${ }^{6}$ E. Vlodarchik and R. Trebinski, Shock Waves 7, 231 (1997).

${ }^{7}$ J. L. Weatherwax, Ph.D. dissertation, MIT, Department of Mathematics, September 2001.

${ }^{8}$ D. S. Drumheller, Introduction to Wave Propagation in Nonlinear Fluids and Solids (Cambridge University Press, Cambridge, U.K., 1998).

${ }^{9}$ J. Smoller, Shock Waves and Reaction-Diffusion Equations (Springer, New York, N.Y., 1983).

${ }^{10} \mathrm{~J}$. L. Weatherwax, D. Vaynblat, O. Bruno, and R. R. Rosales (in progress).

${ }^{11}$ J. M. Hill, One-Dimensional Stefan Problems: An Introduction (John Wiley \& Sons, Inc., New York, 1987).

${ }^{12}$ R. Jeanloz, J. Geophys. Res. 84, 6059 (1979).

${ }^{13}$ D. L. Anderson, Nature 285, 204 (1980).

${ }^{14}$ R. A. Secco, Mineral Physics and Crystallography: A Handbook of Physical Constants (Wiley-Interscience, New York, N.Y., 1995).

${ }^{15}$ W. Band, J. Geophys. Res. 65, 695 (1960).

${ }^{16}$ F. E. Prieto and C. Renero, J. Appl. Phys. 44, 4013 (1973).

${ }^{17}$ A. D. Sakharov, R. M. Zaidel, V. N. Mineev, and A. G. Oleinik, Sov. Phys. Dokl. 9, 1091 (1965).

${ }^{18}$ J. W. Swegle and D. E. Grady, J. Appl. Phys. 58, 692 (1985).

${ }^{19}$ R. D. Richtmyer, Difference Methods for Initial-Value Problems (Interscience, New York, 1967). 